\title{
¿Qué Es una Granja Apoyada por una Alianza de Consumidores? ${ }^{1}$
}

M.E. Swisher, Rose Koenig, Jennifer Gove and James Sterns ${ }^{2}$

\section{Resumen}

Este boletín explica el concepto de una granja apoyada por una alianza de consumadores, una forma de producción en la cual el consumidor se alia directamente con el agricultor para asegurar la producción de sus propios alimentos (Community Supported Agriculture ó CSA en inglés). Aquí se describen los pasos exigidos para desarrollar una alianza de este tipo.

\section{La Granja Apoyada por una Alianza de Consumidores}

Según el Departamento de Agricultura de los Estados Unidos (USDA), una granja apoyada por una alianza de consumidores es una comunidad de individuos ó familias que se comprometen apoyar a un agricultor de tal manera que la granja vuelva la responsabilidad de todos - sea legalmente ó en espíritu. El agricultor y los consumidores se apoyan y comparten los riesgos y los beneficios asociados con la producción de alimentos. Hay bastante otras definiciones de este concepto. Trauger Groh y Steven McFadden, autores de Farms of Tomorrow Revisited señalan que es "una organización de productores y consumidores. Los consumidores se comprometen proveen apoyo directo y en anticipación para los productores locales quienes producen sus alimentos. Los agricultores se comprometen hacer todo lo posible para proveer alimentos de suficiente cantidad y calidad para llenar las necesidades y expectaciones de los consumidores. Hay mucha variación en esta economía asociativa, reflejando los recursos y deseos de los participantes."

Elizabeth Henderson, autor de Sharing the Harvest, explica que una granja de este tipo es "una conexión entre un agricultor local y las personas que consumen los alimentos producidos por él." Robyn Van En dice que es una relación muy sencilla: "agricultores + consumidores + compromiso común = alianza con posibilidades sin límites." Hay varios tipos de organización. "Por un lado existe la alianza que requiere que todos los miembros participan en el trabajo en la granja como obligación. Por otro lado, existe el tipo que se base en una 'subscripción,' en el cual el agricultor y sus empleados se encargan de la producción y los miembros reciben una cantidad fija de alimentos cada semana. La mayoría de las granjas apoyadas por una alianza de

1. This document is FCS7212-Span, one of a series of the Department of Family, Youth and Community Sciences, Florida Cooperative Extension Service, IFAS, University of Florida, Gainesville FL 32611: First published: September 2003. English version reviewed by Gerry Culen, Ph.D. and Amy Simonne, Ph.D., Department of Family, Youth and Community Sciences, and Lisa House, Ph.D., associate professor, Department of Food and Resource Economics, Univeristy of Florida, Gainesville, FL, 32611. Please visit the EDIS Web site at http://edis.ifas.ufl.edu

2. M.E. Swisher, Ph.D., associate professor, Department of Family, Youth and Community Sciences, Rose Koenig, Ph.D., adjunct professor, Department of Plant Pathology, Jennifer Gove, Graduate Assistant, Department of Family, Youth and Community Sciences, and James Sterns, Ph.D., assistant professor, Department of Food and Resource Economics, University of Florida, Gainesville FL 32611. 
consumidores son de tipo intermedio; los miembros trabajan en la finca en ocasiones especiales, apoyan distribuir los productos, ó pagan una parte del costo de ser miembro por trabajo." La Red para la Agricultura Sostenible (Sustainable Agriculture Network) del USDA la define como "una forma más organizada y delimitada del mercadeo por subscripción en la cual los consumidores invierten en la granja por medio de pago adelantado para los alimentos, antes de la cosecha. Los consumidores comparten muchos de los riesgos de la producción agrícola, y también aprovechan de la abundancia de alimentos producidos en los años de alta productividad."

\section{¿Cómo Difiere de la Venta por Subscripción?}

Hay dos diferencias principales entre la venta por subscripción y la formación de una alianza de consumidores y agricultores. Los dos exigen que el consumidor paga en adelante para los productos alimenticios, antes de la cosecha, pero las obligaciones del agricultor varían en los dos casos. En el caso de venta por subscripción, el granjero es obligado entregar una cantidad fija de alimentos a sus clientes. El cliente no tiene ningún riesgo de no recibir los alimentos. Al contrario, el miembro de una alianza de consumidores comparte los riesgos asociados con la producción agropecuaria con el agricultor. El agricultor no tiene ninguna obligación de entregar una cantidad fija de alimentos. Su obligación es sencillamente hacer lo posible para producir una buena cosecha. Además, los dos difieren en cuanto a sus fines. La venta por subscripción es un tipo de mercadeo. Es probable que los clientes tengan mayor confianza con el agricultor que el cliente típico de un supermercado ó un mercado de agricultores - se muestra esta confianza en su voluntad de pagar en adelante para los alimentos. Sin embargo, a base la relación entre el cliente y el agricultor es una relación económica. La confianza entre los agricultores y los consumidores en una alianza se base en los intereses y valores que se comparten. Es algo más que una sencilla relación económica; es una relación social basada, en parte, en una filosofía común del valor de la producción agropecuaria en la vida no solamente de granjeros, sino de consumidores.

\section{Historia}

El desarrollo de granjas apoyadas por alianzas de consumidores empezó en Japón y Europa. El concepto se introdujo en los Estados Unidos in 1986. Actualmente existen aproximadamente 1,000 granjas de este tipo en el país. Indian Line Farm en Massachusetts y Temple-Wilton Community Farm en New Hampshire fueron las primeras granjas que adoptaron esta estrategia en los Estados Unidos en 1986.

\section{Tres Modelos}

Hay tres modelos básicos de granjas apoyadas por consumidores. En el primer modelo el agricultor es el dueño de la tierra, las instalaciones y la maquinaria y él tiene toda la responsabilidad para la producción agrícola. El apoyo de los consumidores consiste de proveer capital por medio de la compra de una "acción" en la alianza. El segundo modelo difiere principalmente en cuanto a la mano de obra. Los consumidores contribuyen una parte importante de la fuerza laboral. En el tercer modelo, realmente no hay un agricultor. Todos los miembros de 
la alianza tienen la responsabilidad para la producción y ellos mismos, como grupo, son los dueños de los medios de producción.

\section{¿Por qué los Consumidores Participan en estas Alianzas?}

Los consumidores tienen razones diferentes para participar en una alianza. Algunos prefieren los alimentos producidos localmente. Se reducen los costos y los daños ocasionados por el transporte de productos frescos sobre largas distancias. Además, el dinero pagado para los productos se invierte en la comunidad local. Otra razón importante para muchos consumidores es la habilidad de conseguir productos alimenticios no disponibles en los supermercados, tales como productos de poca demanda ó aquellos que se dañan fácilmente por el transporte. Consumidores participan porque quieren apoyar a los agricultores locales, obtener productos frescos y de alta calidad, reducir el costo de los alimentos y preservar el paisaje rural y el medio ambiente. Para muchos consumidores, la relación directa con la producción agropecuaria es también de alta importancia. Les gusta entender como se producen sus alimentos.

\section{El Establecimiento de una Alianza}

Según Henderson y Van En, la decisión de establecer una alianza entre consumidores y agricultores es "un proceso llevado a cabo por un grupo dedicado de consumidores quienes quieren compartir los riesgos y los beneficios de la producción agropecuaria con los granjeros. Como el agricultor tiene un mercado asegurado para sus productos e ingresos ciertos, él puede fijarse en la producción de alimentos de alta calidad y la protección de la tierra. Los miembros disfrutan de los alimentos más frescos, más sabrosos y más nutritivos que se han gustado en toda la vida, como si tuviesen sus propios huertos, pero con mucha menos labor." Los agricultores experimentados, quienes ya tienen su terreno y maquinaria y han desarrollado un grupo de clientes fieles, lo encuentran más fácil formar una alianza con consumidores. Los agricultores jóvenes ó de poca experiencia encuentran mayores retos. Sin embargo, muchos agricultores por todo el país han logrado éxito en establecer alianzas con consumidores.

No existe una "receta" para el establecimiento de una alianza de consumidores y agricultores. No obstante, se puede notar unos pasos fundamentales para el éxito.

\section{Organización inicial de un grupo sólido de partidarios}

Una sola persona no puede desarrollar una alianza exitosa, ni el agricultor más experimentado, ni el consumidor más dedicado. La identificación de un pequeño grupo de individuos dedicados al concepto de establecer una alianza es crítica. En algunos casos, son los consumidores que empiezan el proceso, en cual caso ellos tienen que identificar uno o más agricultores dispuestos a participar en la alianza. En otros casos, la idea surge del agricultor. En cualquier caso, el punto clave es encontrar una media docena de personas dispuestas a invertir el tiempo y esfuerzo necesarios para hacer una realidad de la alianza. 


\section{Estructura y responsabilidades}

En los Estados Unidos, la mayoría de las granas apoyadas por alianzas de consumidores se basen en granjas existentes. Hay relativamente pocos casos en este país en los cuales los miembros mismos son los dueños de la granja y lo manejan directamente. Obviamente, el tercer modelo de organización requiere altos niveles de consonancia entre los miembros de la alianza. Sin embargo, casi todas las alianzas tienen un grupo central ó un núcleo de miembros (elegidos ó autoseleccionados por su interés) quienes asumen mucha de la responsabilidad para el manejo de la alianza. Se requiere un entendimiento cierto de las obligaciones y responsabilidades de todos los miembros y especialmente de las del agricultor y del núcleo. Generalmente, el agricultor mismo prefiere hacer las decisiones cotidianas. No obstante, es necesario llegar a un acuerdo en cuanto a muchos otros factores. ¿Quién decidirá qué sembrar? ¿Quién decidirá la cantidad de alimentos proveídos a cada miembro? ¿Cómo se comunicarán los miembros? ¿Quién organizará las reuniones del grupo? ¿Cómo se repartirán los productos y quién tendrá la responsabilidad para hacerlo, el agricultor, el grupo central, ó los miembros mismos? Sin una buena repartición de las responsabilidades y del trabajo, se desarrollan las quejas y la alianza se vuelve una complicación más en la vida, tanto para el agricultor como para los consumidores.

\section{Reclutamiento de miembros}

Las alianzas exitosas generalmente empiezan en pequeña escala. Plowshares de Gainesville, Florida, empezó con unos 20 miembros, por ejemplo. Como cualquiera otra organización, una alianza de este tipo encuentra retos y problemas inicialmente. Es mucho más fácil resolverlos cuando el grupo es pequeño. El reclutamiento inicial es casi siempre por contacto personal. Los clubes sociales, las iglesias, y las empresas son fuentes de nuevos miembros. Sobre tiempo, la alianza crece naturalmente si se logra producir alimentos de alta calidad para sus miembros.

\section{¿Qué es que el agricultor necesita saber?}

Como el proveedor de una buena parte de los alimentos para muchas familias, el agricultor tiene que producir una diversidad de productos. La mayoría de las alianzas se enfocan en la producción hortícola, pero también existen algunas enfocadas en la producción pecuaria. En ambos casos, los miembros de la alianza dependen del agricultor para llenar la mayoría de sus necesidades. Obviamente, no quieren consumir la misma comida todos los días y una de las quejas más comunes de los consumidores en estos grupos es que la variedad de alimentos no es suficiente. Es altamente importante ofrecer a los consumidores una variedad de productos cada semana y de variar los productos a lo largo del año. En general, los consumidores también buscan alta calidad y alimentos novedosos. El agricultor exitoso identifica fuentes de cultivos nuevos y variedades con calidades especiales, tales como un color diferente (la papa morena) ó un sabor particular (las viejas variedades de tomate). Así, se puede mantener el interés de los consumidores y evitar el problema de perder miembros.

Las otras habilidades son de igual importancia. La constancia de la producción y la distribución es de primordial importancia para los miembros del grupo. Aunque los consumidores comparten los riesgos con el agricultor, el agricultor tiene la responsabilidad de proveer los alimentos con regularidad. En la mayoría de las alianzas, es el agricultor que tiene la principal responsabilidad para la coordinación de las actividades cotidianas de la alianza, incluyendo la mano de obra de los miembros y la repartición de los alimentos. También es normal que el agricultor se encarga de la planificación, incluyendo el desarrollo del presupuesto y el manejo de las cuentas de la granja. 
Aunque la alianza se base en la confianza entre los miembros, el agricultor tiene que preocuparse por muchos asuntos legales, tales como seguros. Además, el agricultor exitoso es generalmente una persona con buena experiencia en el mercadeo y una persona que se lleva bien con otros, capaz de mantener buenas relaciones humanas.

\section{La Junta Directiva}

El núcleo de cualquiera alianza es la junta directiva. Este grupo, que incluye consumidores y el agricultor, es responsable para el manejo de sistema de producción y la repartición de alimentos. La junta directiva es elegida en algunos casos, pero muchas veces es auto-seleccionada, basada en el interés y el tiempo disponible a los miembros. Generalmente, la junta directiva tiene seis responsabilidades principales:

Decidir cuales cultivos el agricultor debe sembrar

Determinar el costo de participación y cuando se debe pagar

Seleccionar uno ó más agricultores (si no hay un agricultor en el grupo original)

Si es necesario, buscar el terreno para la granja

Asignar responsabilidades a los demás miembros

Decidir como distribuir los alimentos

Las actividades de la junta directiva de Plowshares en Gainesville, Florida, son típicas. La junta se encuentra cada mes. Se revisan las actividades agrícolas y las cuentas del grupo. Los miembros de la junta divulgan un folleto que describe la alianza y una carta informativa para los miembros, organizan actividades especiales tales como festivales y manejan el reclutamiento de miembros y la organización general del grupo.

En muchas alianzas, los diferentes miembros de la junta asumen responsabilidades específicas. Muchas alianzas identifican un coordinador de socios y un tesorero. El coordinador de socios asegura la comunicación entre el agricultor y los miembros, incluso hacer notar inquietudes y problemas. Se encarga de la correspondencia del grupo y generalmente toma la responsabilidad para la colecta de las cuotas. El tesorero maneja las cuentas del grupo. Generalmente esta persona recibe las cuotas recogidas por el coordinador de socios, las registra y deposita en el banco y al fin distribuye la capital al agricultor. Otros puestos comunes incluyen los coordinadores de comunicaciones, de voluntarios y de actividades sociales. Muchas alianzas donan alimentos a familias pobres. En unos casos, es una donación semanal. En otros casos, se donan los alimentos que los miembros no recogen (cuando están en vacaciones, etc.). Por lo tanto, unas alianzas también identifican un coordinador y de apoyo para familias de pocos recursos.

\section{Los Fundamentos}

Costo. El costo de ser miembro de una alianza varía mucho y se base en muchos factores, entre otros los costos de producción, el valor de los alimentos en los supermercados, la cantidad de alimentos proveídos al consumidor cada semana y la temporada productiva cada año. Unas alianzas ofrecen precios reducidos para familias humildes. Otras permiten a los miembros pagar una parte de la cuota con mano de obra.

Diversificación de Productos. Es casi imposible que una sola granja produce todos los productos que los miembros de una alianza quieren recibir. Por lo tanto, unas alianzas complementan los alimentos producidos en la granja con otros obtenidos de otras fuentes, lo cual aumenta la diversidad de productos disponibles a los miembros y reduce el riesgo de quedarse sin 
alimentos en caso de una situación improvista. Sin embargo, también hay desventajas, incluyendo mayor mano de obra, mayores gastos y complicaciones en el manejo de las cuentas. Otros grupos ofrecen mayor diversidad a sus miembros por medio de distribuir los alimentos en localidades donde otras comidas son disponibles, tales como un mercado del agricultor ó una cooperativa.

Distribución. Es normal distribuir los alimentos semanalmente. Muchas alianzas distribuyen los alimentos dos días a la semana para ofrecer mayor flexibilidad a los miembros. Unas permiten que los miembros recolectan sus alimentos en la finca misma, pero muchas utilizas otros sitios, tales como cooperativos y mercados. Unas entregan los alimentos en la casa misma del consumidor (generalmente por un costo adicional) y algunas los envían por correo.

Pago por Mano de Obra. Unas alianzas permiten que los miembros pagan una parte de la cuota por mano de obra. Antes de decidir si esta política es apropiada para una alianza, se debe considerar tanto las necesidades del agricultor y de la alianza como aquellas de los miembros. Unos agricultores prefieren no tener muchos trabajadores en sus granjas por varias razones, incluso la responsabilidad legal. En muchos casos los consumidores consisten principalmente de familias en las cuales los dos padres trabajan tiempo completo y no tienen el tiempo disponible regularmente para trabajar en la finca. Es importante establecer claramente el valor de la mano de obra si se decide permitir pago por mano de obra para evitar malentendidos.

Voluntarios. Muchas alianzas ocupan de voluntarios ocasionalmente ó con regularidad. El papel de los voluntarios varía mucho, desde mano de obra para las actividades productivas hasta apoyo en ocasiones especiales, tales como festivales. Se debe pensar bien antes de aceptar voluntarios. ¿Cuántos se necesitarán? ¿Cuándo? ¿Habrá responsabilidades legales asociadas con su presencia en la finca? ¿Se permitirán los jóvenes de menos de 18 años trabajan en la granja? ¿Si se permiten, se necesitará supervisión por adultos?

Reclutamiento y Retención de Miembros. Un folleto ó boletín que describe la alianza es fundamental para el reclutamiento (ver ejemplo en esta publicación). El folleto debe indicar el costo de ser miembro, cuando se debe pagar, cuando y donde se puede recoger los alimentos, la temporada productiva y una aproximación de la cantidad de alimentos que el consumidor puede esperar cada semana. También se debe describir la granja, los métodos de producción (orgánica, por ejemplo) y los alimentos producidos en cada época del año. La mayoría de los folletos incluyen el formulario que se debe entregar para hacerse miembro y una descripción de otros aspectos interesantes de la alianza, tales como festivales o actividades sociales. Siempre se perderán unos miembros, pero es importante contactar los miembros que abandonan la alianza para saber las razones para desligarse de la alianza. Además, si se sabe el número de miembros que se pierden cada año, es más fácil desarrollar una estrategia de reclutamiento para mantener un número constante de miembros.

Comunicación con los Miembros. Es absolutamente necesario mantener la comunicación con los miembros del grupo. La mayoría de alianzas hacen una encuesta de los miembros cada año, generalmente al fin de la temporada productiva, aunque algunas hacen dos encuestas, una durante la temporada y otra al fin. Casi siempre se incluyen preguntas sobre la cantidad y calidad de los alimentos ofrecidos, las preferencias de los consumidores en cuanto a los alimentos presentados y sus opiniones del provecho de ser miembro. Además que encuestas formales, la junta directiva siempre debe mantener contacto directo con los miembros y escucha bien sus opiniones e inquietudes. Muchas alianzas divulgan una carta informativa, una buena media de comunicación regular con los miembros. Es necesario determinar la frecuencia de publicación y quienes se encargarán de publicarla. 
Responsabilidades Legales. Henderson y Van En describen las responsabilidades legales relacionadas al desarrollo de una alianza: "La mayoría de alianzas están asegurados por la responsabilidad civil. Este tipo de seguro puede ser muy costoso si se lo compra por aparte, pero se reduce el costo cuando se lo incluye como un elemento en una póliza total para una granja. Se debe intentar conseguir una póliza que permite pagar una cuota fija y específica para gastos medicales sin tener que presentar una demanda a un tribunal. Las alianzas que permiten a los miembros trabajar en la granja afrontan riesgos legales adicionales y se debe conseguir una póliza que incluye protección para estos tipos de actividades. Se puede reducir el costo de este tipo de seguro si se evite el uso de plaguicidas sintéticas y si los miembros no utilizan maquinaria ó escaleras y no se montan en caballos. Generalmente la póliza permite a los miembros utilizar herramientas y participar en la cosecha. En todo caso, siempre se debe tener un botequín de emergencia fácilmente disponible."

Henderson y Van En también hablan de la estructural legal de las alianzas: "Las alianzas utilizan una variedad de estructuras legales. Cada grupo debe identificar la forma más apropiada para sus condiciones. Algunas alianzas son consideradas como la propiedad de un individuo ó una sociedad en comandita; es decir, tanto la granja como la alianza, considerada como un negocio, son la propiedad del agricultor. Otros grupos distinguen entre el propietario de la granja ó de la tierra y él del negocio o alianza. Un individuo, una sociedad en comandita o una sociedad anónima puede ser el dueño de la tierra, mientras que la alianza se constituye una entidad no incorporada o una sociedad anónima sin fines de lucro. Los agricultores también puede organizarse en grupos para formar cooperativas, la mayoría de las cuales son sociedades anónimas. No hay una estructura única dictada por la ley en cuanto a la forma legal para cooperativas de consumidores; por lo tanto los consumidores tienen la oportunidad de cambiar la estructura de la alianza para mejor cumplir con sus propios fines... Cada estructura legal tiene ventajas y desventajas. Los detalles legales varían en los diferentes estados [de los EEUU]."

Integración en la Comunidad. La filosofía de las alianzas de este tipo enfatiza la importancia de participar en la comunidad y especialmente en los programas sociales locales. Cada grupo tiene que identificar las oportunidades de integrarse en la vida comunal que se cumplen mejor con los objetivos y metas del grupo.

\section{Recursos}

Appropriate Technology Transfer for Rural Areas (ATTRA)

Alternative Farming Information Center - National Agriculture Library Research Service

The Biodynamic Farming and Gardening Association

Sustainable Agriculture Network

The Center for Sustainable Living - Robyn Van En Center

Southern Sustainable Agriculture Working Group

\section{Libros y Boletines}

Sharing the Harvest - Elizabeth Henderson and Robyn Van En

Farms of Tomorrow Revisited - Groh and McFadden

From Asparagus to Zucchini: A Guide to Farm Fresh Seasonal Produce-Madison Area

Community Supported Agriculture Coalition

Growing for Market: News and Ideas for Market Gardeners: Fairplain Publications

The Community Farm: A Voice for CSA 


\section{Referencias}

Groh, T., \& McFadden, S. (1997). Farms of Tomorrow Revisited: Community Supported Farms, Farm Supported Communities. Kimberton, PA: Biodynamic Farming and Gardening Association.

Henderson, E., \& Van En, R. (1999). Sharing the Harvest. White River Junction, VT: Chelsea Green Publishing Company.

Sustainable Agriculture Network, United States Department of Agriculture. (1999). Marketing

Strategies for Farmers and Ranchers. (SAN Publication no. 11/99). Washington, DC: Sustainable Agriculture Network. 


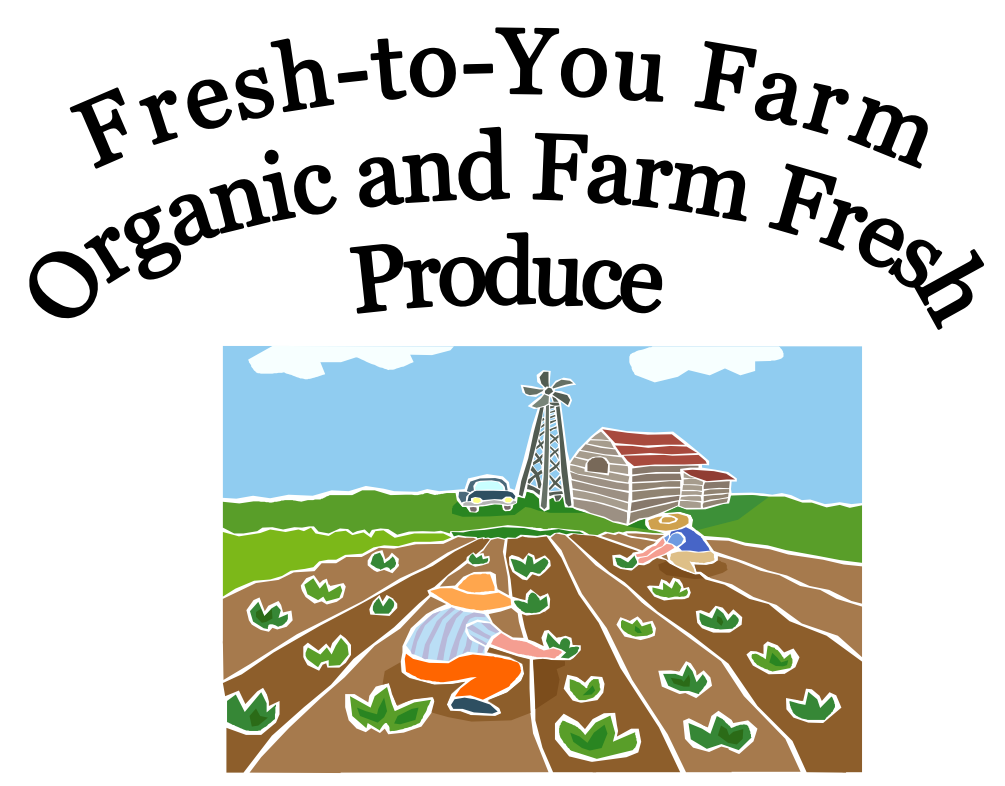

123 Main Street

Yourtown, Florida

32307

(356)987-6543 Today!

Now Accepting Membership Applications! Fill Out Your Application on the Back of this Brochure

Organically grown
vegetables
10 month
season: October
through June
Flexible pickup
schedule
Payment options
Fresh cut flowers
Fresh cut herbs

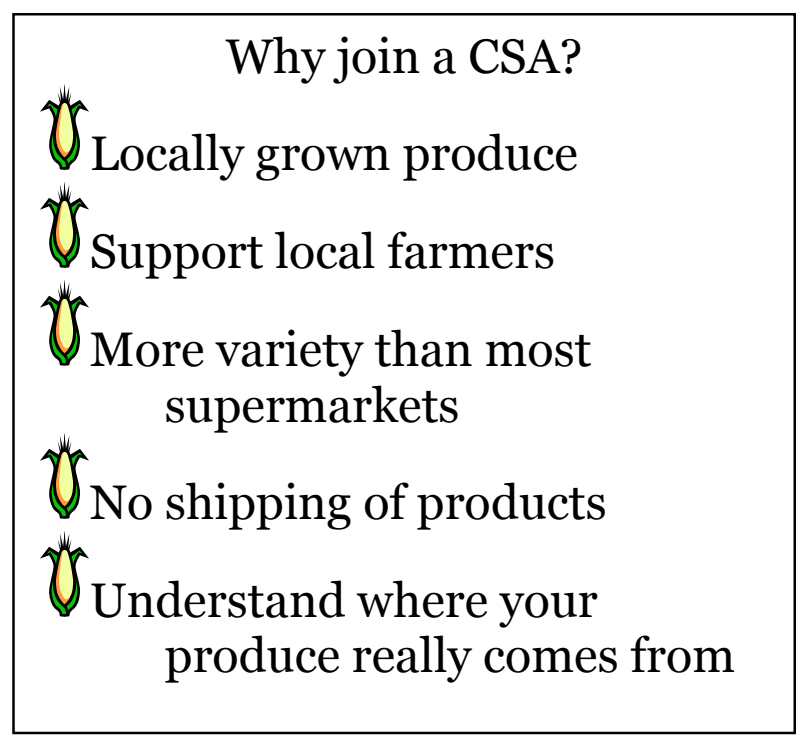

What is CSA? CSA is community supported agriculture that allows the customer direct access to farm fresh produce as well as the opportunity to learn where his or her food comes from and to participate in farming activities.

For more information or a tour of the farm, call (356)987-6543. 


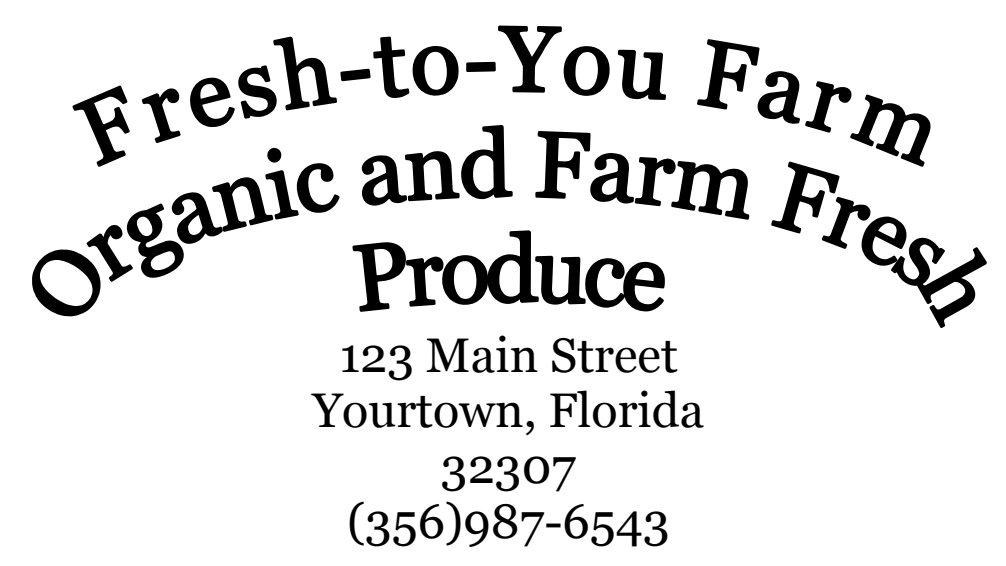

Name:

Address:

Phone (days): (evenings)

Email address: Fax:

I would like to purchase: One full share (\$475) One half share $(\$ 290)$ 4 Week Trial (\$115)

Payment Options:

Check or money order for full amount now

Check or money order for $\$ 50$ now and remaining balance will be paid in full by Oct. 15

I would prefer pickup on:

Monday Tuesday Thursday I'm flexible

The following people are allowed to pick up my share if I cannot do so:

I understand that there will be no refunds given after November $1^{\text {st }}$. I also understand that each week my share will include different types and amounts of produce. I understand that if I do not pick up my share myself or it is not picked up by someone who is authorized to do so by the end of the designated pick up time, that my share will be donated to a worthy recipient.

\section{Date}

